\title{
Strong Electronic Correlations in a New Yb-Based Compound: $\mathrm{YbCu}_{4} \mathrm{Ni}$
}

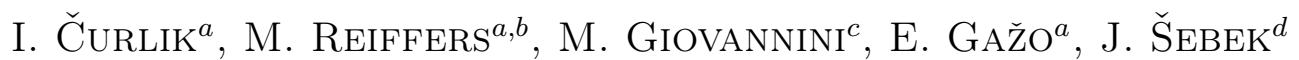 \\ AND E. ŠSANTAVÁ ${ }^{d}$ \\ ${ }^{a}$ Institute of Experimental Physics, Watsonova 47, SK 04353 Košice, Slovakia \\ ${ }^{b}$ Faculty of Sciences, University of Prešov, 17. novembra 1, SK 08078 Prešov, Slovakia \\ ${ }^{c}$ CNR-SPIN and Department of Chemistry, University of Genova, I-16146 Genova, Italy \\ ${ }^{d}$ Institute of Physics, V Holešovičkách 2, CZ-18000 Praha, Czech Republic
}

\begin{abstract}
Heat capacity measurements performed on the new ternary compound $\mathrm{YbCu}_{4} \mathrm{Ni}$ indicate for this compound strong electronic correlations with possible antiferromagnetic phase transition below 0.5 K. Susceptibility and magnetisation measurements above $2 \mathrm{~K}$ show no magnetic ordering.
\end{abstract}

PACS numbers: 71.27.+a, 75.30.-m, 75.30.Mb

\section{Introduction}

Strong correlation between electrons, due to hybridization of $f$-electrons and conduction electrons, can cause a number of outstanding low temperature features. Among the rare earths, a large number of these phenomena is found for Ce- and Yb-based compounds [1].

Recently a strong emphasis has been given to the investigation of the solid solution $\mathrm{YbCu}_{5-x} \mathrm{M}_{x}(\mathrm{M}=\mathrm{Ag}$, $\mathrm{Au}, \mathrm{In})$ crystallizing in the cubic $\mathrm{AuBe}_{5}$-type structure, where the substitution of $\mathrm{Cu}$ by $\mathrm{M}$ offers the possibility to study the different evolution of ground state properties depending on the $\mathrm{M}$ element $[2,3]$. Very recently, a competition in compound formation between $\mathrm{Yb}(\mathrm{Cu}, \mathrm{T})_{5}$ and its monoclinic superstructure $\mathrm{Yb}(\mathrm{Cu}, \mathrm{T})_{4.5}$ was revealed [4]. The interest in this topic was initially triggered by the investigation on the heavy fermions $\mathrm{YbCu}_{4} \mathrm{~T}$ $(\mathrm{T}=\mathrm{Ag}, \mathrm{Au})$, which crystallize in the cubic $\mathrm{MgCu}_{4} \mathrm{Sn}$ type (an ordered derivative of the $\mathrm{AuBe}_{5}$-type). It was found that $\mathrm{YbCu}_{4} \mathrm{Au}$ orders magnetically below $1 \mathrm{~K}$, whereas $\mathrm{YbCu}_{4} \mathrm{Ag}$ has a nonmagnetic ground state [5].

In this work results on $\mathrm{YbCu}_{4} \mathrm{Ni}$, a new member of the family $\mathrm{YbCu}_{4} \mathrm{~T}(\mathrm{~T}=$ transition metal), are presented. A competition on compound formation between the cubic and its monoclinic superstructure was found also for this system. Nevertheless, our attempt to prepare a sample with a cubic structure (lattice parameter $a=6.9429 \AA$ ) was successful. Furthermore, a Rietveld refinement carried out on the sample prepared shows a very good agreement with the model of the $\mathrm{MgCu}_{4} \mathrm{Sn}$ structure type with a full ordered distribution of the atoms at the sites of the structure (the details on the characterization, compound formation and the crystal data will be shown elsewhere). We present the results of study of thermal and magnetic properties in the temperature range $0.3-300 \mathrm{~K}$ and in an applied magnetic field up to $9 \mathrm{~T}$.

\section{Experimental}

The polycrystalline samples of $\mathrm{YbCu}_{4} \mathrm{Ni}$ have been prepared by weighting the stoichiometric amount of elements with the following nominal purity: $\mathrm{Yb}(99.9 \%$ mass), $\mathrm{Cu}$ (99.999\% mass) and $\mathrm{Ni}(99.995 \%$ mass). The elements were enclosed in small tantalum crucibles, sealed by arc welding under pure argon, in order to avoid the loss of $\mathrm{Yb}$ with a high vapour pressure. The samples were then melted in an induction furnace, under a stream of pure argon. To ensure homogeneity during the melting, the crucibles were continuously shaked. The samples were then annealed in a resistance furnace at $700^{\circ} \mathrm{C}$ for two weeks and finally quenched in cold water. The crystalline structure was examined by X-ray diffraction. Heat capacity measurements were performed by PPMS commercial device (Quantum Design) in the temperature range $0.3-300 \mathrm{~K}$ and in an applied magnetic field up to 9 T. Heat capacity was measured using the two- $\tau$ model of the relaxation method. Susceptibility (at excitation field $1600 \mathrm{~A} / \mathrm{m}$ ) and magnetisation were measured by MPMS commercial device (Quantum Design) in the temperature range $2-300 \mathrm{~K}$ and in an applied magnetic field up to $5 \mathrm{~T}$.

\section{Results and discussion}

Figure 1 shows the low temperature dependence of the heat capacity $C(T)$ of $\mathrm{YbCu}_{4} \mathrm{Ni}$ measured for various values of applied magnetic fields up to $9 \mathrm{~T}$. By increasing 
magnetic fields, up $0.3 \mathrm{~T}$ we observed at $0.5 \mathrm{~K}$ a small sharp anomaly of increasing intensity. With an applied magnetic field of $0.6 \mathrm{~T}$, a broader maximum appears, which shifts to higher temperatures and broadens with increasing magnetic field, like a Schottky anomaly. This behaviour could be explained by the lifting of the degeneracy of the $\mathrm{Yb}$ crystalline electric field (CEF) ground-state doublet with increasing magnetic field, due to the Zeeman splitting. Correspondingly, the associated Schottky anomaly is shifted to higher temperatures, similarly to the case of the isotypic $\mathrm{YbCu}_{4} \mathrm{Au}$ [6]. Moreover, a small hump at $2.5 \mathrm{~K}$, which is quenched in magnetic fields above $0.3 \mathrm{~T}$, reflects a low amount of $\mathrm{Yb}_{2} \mathrm{O}_{3}$ in the sample [7]. The sharp anomaly at low applied magnetic field could be explained as possible indication of an expected antiferromagnetic phase transition below $0.5 \mathrm{~K}$, similarly to the situation observed in $\mathrm{YbCu}_{4} \mathrm{Au}[6]$. However, measurements in the millikelvin temperature range are required.

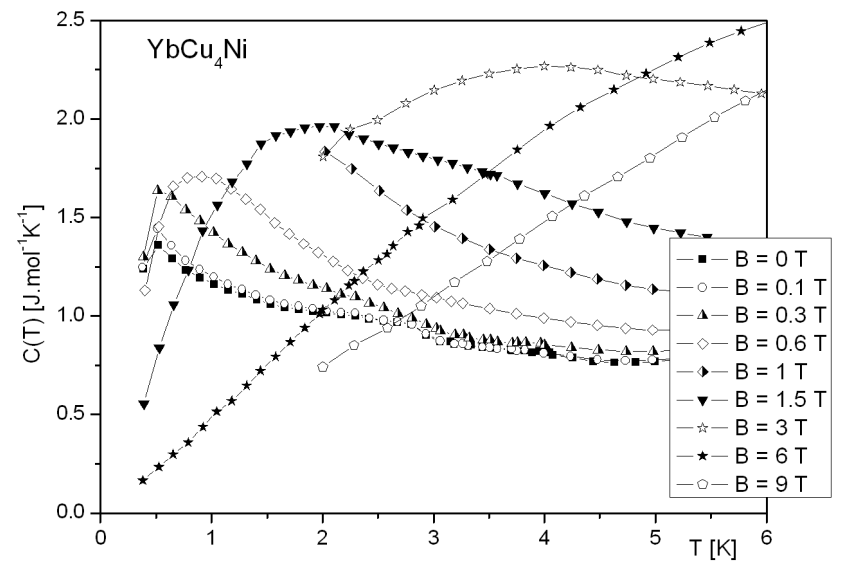

Fig. 1. Low temperature detail of dependence of the heat capacity $C(T)$ of $\mathrm{YbCu}_{4} \mathrm{Ni}$ as function of applied magnetic field.

In order to determine the electronic $\gamma$ coefficient we analysed the dependence of $C / T$ as a function of $T^{2}$. If we take the extrapolation in the temperature range above $6.5 \mathrm{~K}$, we obtain $\gamma=110 \mathrm{~mJ} /\left(\mathrm{mol} \mathrm{K}{ }^{2}\right)$. However, below these temperatures $C / T$ begins to diverge to higher values which could be connected with a possible magnetic phase transition at $T_{\mathrm{N}}$. Similarly to the behaviour of $\mathrm{YbCu}_{4} \mathrm{Au}$ [6], the applied magnetic field should suppress $T_{\mathrm{N}}$. At $9 \mathrm{~T}$ we obtained by extrapolation $\gamma=370 \mathrm{~mJ} /\left(\mathrm{mol} \mathrm{K}^{2}\right)$, which is an indication of heavy fermion behaviour.

Figure 2 shows the temperature dependence of the inverse magnetic susceptibility $\chi^{-1}(T)$ of $\mathrm{YbCu}_{4} \mathrm{Ni}$. The compound exhibits simple (with negligible small $\chi_{0}$ contribution) Curie-Weiss behaviour above $100 \mathrm{~K}$. From $\chi^{-1}(T)$ dependence we have determined the paramagnetic Curie temperature $\Theta_{\mathrm{P}}=-18.6 \mathrm{~K}$. This supports the possible existence of an antiferromagnetic phase transition at very low temperatures. From the Curie constant the effective magnetic moment $\mu_{\mathrm{eff}}=4.8 \mu_{\mathrm{B}}$ was obtained, which is quite close to the free ion value $4.54 \mu_{\mathrm{B}}$.

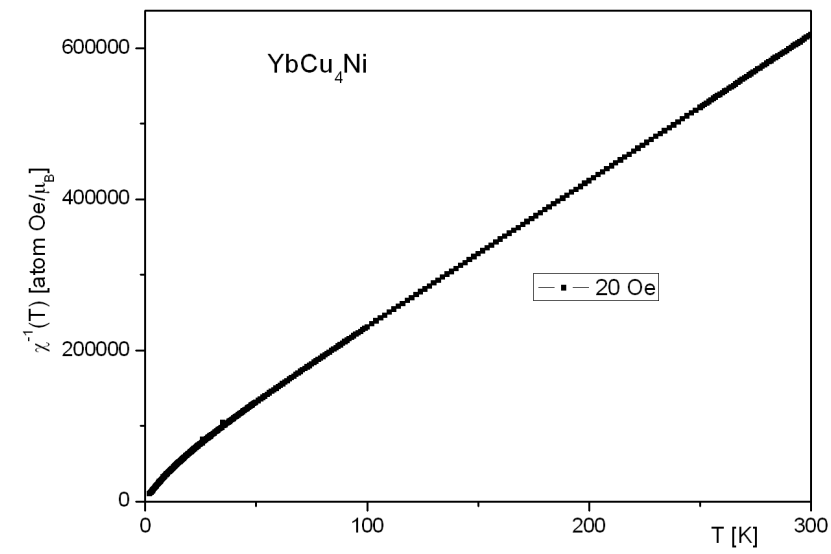

Fig. 2. Temperature dependence of the inverse susceptibility $\chi^{-1}(T)$ of $\mathrm{YbCu}_{4} \mathrm{Ni}$.

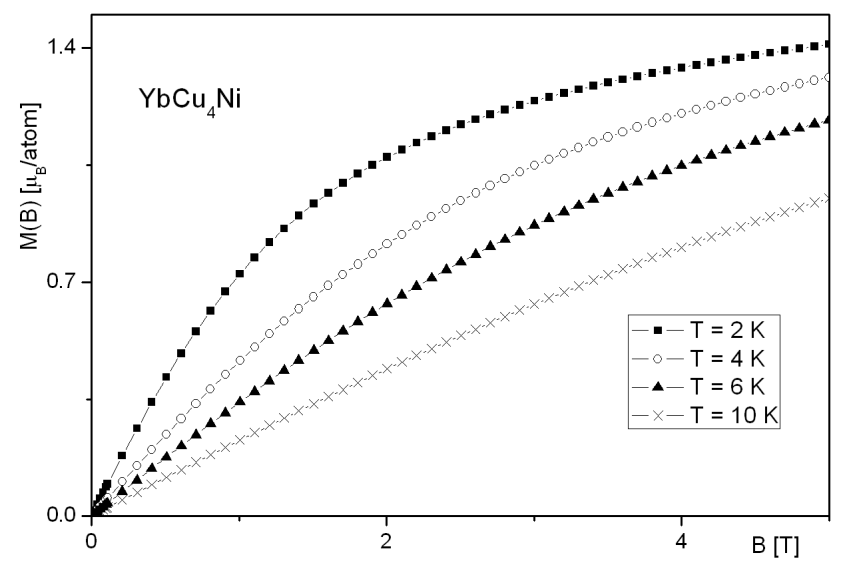

Fig. 3. The field dependence of the magnetization $M(B)$ of $\mathrm{YbCu}_{4} \mathrm{Ni}$.

Figure 3 shows the magnetic field dependence of magnetisation $M(B)$ of $\mathrm{YbCu}_{4} \mathrm{Ni}$ for different temperatures. The dependences are characteristic for paramagnetic behaviour with a tendency to saturation at lower temperatures. Therefore our data support the fact that $\mathrm{YbCu}_{4} \mathrm{Ni}$ is a new strongly electron correlated compound. Further measurements in the lower temperature range below $0.5 \mathrm{~K}$ are required in order to study the possible magnetic phase transition.

\section{Conclusion}

In conclusion, a novel $\mathrm{YbCu}_{4} \mathrm{Ni}$ compound with strong electronic correlation, which crystallizes in the cubic $\mathrm{MgCu}_{4} \mathrm{Sn}$ type, was prepared. Heat capacity $C(T)$ up to an applied magnetic field of $9 \mathrm{~T}$, susceptibility $\chi(T)$ and magnetisation $M(B)$ up to $5 \mathrm{~T}$, were measured. We determined an electronic coefficient $\gamma=$ 
$110 \mathrm{~mJ} /\left(\mathrm{mol} \mathrm{K}^{2}\right)$ which changes upon magnetic field $9 \mathrm{~T}$ to $370 \mathrm{~mJ} /\left(\mathrm{mol} \mathrm{K}^{2}\right)$; Curie temperature $\Theta_{\mathrm{P}}=-18.6 \mathrm{~K}$ and effective magnetic moment $\mu_{\mathrm{eff}}=4.8 \mu_{\mathrm{B}}$.

\section{Acknowledgments}

This work has been partly supported by the SlovakItalian Scientific-Technological Exchange Program for fellowships in Genova and Kosice; Slovak Research and Development Agency under the contract No. APVV-SK-IT-0023-08 and VVCE-0058-07; the Slovak grant agency VEGA $2 / 0007 / 09$; the CLTP as the Centre of Excellence of the Slovak Academy of Sciences and P.J. Safárik University; the CEX Nanofluid as the Centre of Excellence SAS and by 7th FP EU MICROKELVIN. J. ̌́. and E.S. acknowledge for the support GAČR 202/09/0030. The liquid nitrogen for the experiment has been sponsored by the U.S. Steel Kosice, s.r.o.

\section{References}

[1] M. Giovannini, E. Bauer, H. Michor, G. Hilscher, A. Galatanu, A. Saccone, P. Rogl, Intermetallics $\mathbf{9}$, $481(2001)$

[2] N. Tsujii, J. He, K. Yoshimura, K. Kosuge, H. Michor, K. Kreiner, G. Hilscher, Phys. Rev. B 55, 1032 (1997).

[3] M. Giovannini, A. Saccone, St. Muller, H. Michor, E. Bauer, J. Phys., Condens. Matter 17, S877 (2005).

[4] M. Giovannini, R. Pasero, S. De Negri, A. Saccone, Intermetallics 16, 399 (2008).

[5] C. Rossel, K.N. Yang, M.B. Maple, Z. Fisk, E. Zirngiebl, J.D. Thompson, Phys. Rev. B 35, 1914 (1987).

[6] E. Bauer, E. Gratz, R. Hauser, Le Tuan, A. Galatanu, A. Kottar, H. Michor, W. Perthold, G. Hilscher, T. Kagayama, G. Oomi, N. Ichimiya, S. Endo, Phys. Rev. B 50, 9300 (1994).

[7] H. Nowatari, Y. Saiga, Y. Kato, K. Iwata, S. Katano, T. Fujiwara, Y. Uwatoko, M. Kosaka, J. Phys. Soc. Jpn. 76, suppl. A, 80 (2007). 